Short communication

\title{
The drag coefficient of a sphere: An approximation using Shanks transform
}

\author{
M.D. Mikhailov ${ }^{a}$, A.P. Silva Freire ${ }^{\text {b,* }}$ \\ a Scientific Division, Brazilian National Institute of Metrology (DINAM/DIMCI/INMETRO), 22050-050, Rio de Janeiro, Brazil \\ b Mechanical Engineering Program (PEM/COPPE/UFRJ), C.P. 68503, 21941-972, Rio de Janeiro, Brazil
}

\section{A R T I C L E I N F O}

\section{Article history}

Received 19 September 2012

Received in revised form 5 December 2012

Accepted 15 December 2012

Available online 22 December 2012

Keywords:

Sphere drag

Oseen flow

Shanks transform

\begin{abstract}
A B S T R A C T
An accurate model for the drag coefficient $\left(C_{D}\right)$ of a falling sphere is presented in terms of a non-linear rational fractional transform of the series of Goldstein (Proc. Roy. Soc. London A, 123, 225-235, 1929) to Oseen's equation. The coefficients of the six polynomial terms are improved through a direct fit to the experimental data of Roos and Willmarth (AIAA J., 9:285-290, 1971). The model predicts $C_{D}$ up to Reynolds number 100,000 with a standard deviation of 0.04. Results are compared with eight different formulations of other authors.
\end{abstract}

(c) 2012 Elsevier B.V. All rights reserved.

\section{Introduction}

The case of a free falling (or rising) sphere with constant velocity in an unbounded environment is relevant to numerous practical problems. Many applications in chemical and metallurgical processes, in sediment transport and deposition in channels and pipes require the specification of the drag coefficient and the settling velocity of spherical bubbles, drops or particles.

Unfortunately, the Navier-Stokes (N.-S.) equations do not exhibit exact solutions for flows around bodies of finite size, for any range of particle Reynolds number $\left(R_{p}\right)$. At some extreme values of $R_{p}$, approximate analytical methods can be used to derive equations that yield useful approximate solutions. For a spherical particle, these are frequently limited to $R_{p}<30$ (Liao [1]).

In view of the above remarks, expressions for the drag coefficient of a sphere $\left(C_{D}\right)$ with a large interval of application need to be obtained from empirical or numerical data through regression techniques. The various approximations quoted in literature vary somewhat in form (Cheng [2]), but normally are expressed in terms of single power series expansions on $R_{p}$. Piecewise or asymptotically matched small segments (Almedeij [3]) are also frequently used. Here, an approach is proposed to determine an empirical expression for the drag coefficient of a sphere based on Shanks transform [4]. This is a non-linear transform that is very effective to accelerate the convergence of slowly converging series.

\footnotetext{
* Corresponding author.

E-mail addresses: atilafreire@gmail.com, atila@mecanica.coppe.ufrj.br (A.P.S. Freire).
}

Shanks very early recognized the advantages of working with rational fractions of higher order provided more than three terms of a power series are known. For a falling sphere, his proposed 6-term expression for the drag coefficient based on the first five terms of Goldstein's series (see Eq. (6) below) agrees well with experiments up to $R_{p}=10$. An increase on the number of terms retained by a Shanks transformation does not improve predictions. For example, a 16-term expression diverges strongly for $R_{p}>10$.

In the present work, the generalized transformation of Shanks initially applied to the 6-term extended solution proposed by Goldstein [5] is fitted directly to the data of Roos and Willmarth [6]. The resulting rational fractional formula is valid up to the critical range of $R_{p}$, with a standard deviation of 0.04 based on the difference between the computed and the reference values.

The expression that results from the data of Roos and Willmarth [6] is valid in the interval $5.33<R_{p}<118,300$. For $R_{p}$ below this value, the expression of Shanks [4] should be used. Alternatively, however, we have considered some extra experimental points extracted from Brown and Lawler [7] to improve results for low $R_{p}$. This other expression offers slightly higher values of $C_{D}$ for very high $R_{p}$ numbers.

A comparison between the present results and the different formulations reviewed in Cheng [2] is also presented.

\section{The solutions of Stokes and of Oseen}

This section briefly reviews the solutions of Stokes [8] and Oseen $[9,10]$, that have served as a standing block to the solutions of Goldstein [5] and Shanks [4] (see also Van Dyke [11]).

For very small $R_{p}$, Stokes [8] neglected the inertia terms in the N.-S. equations to find, for a steady streaming motion, the drag expression, $D=6 \pi \mu U a$, where $a$ is the radius of the sphere, $U$ is the free stream 
velocity and $\mu$ is the viscosity of the fluid. The corresponding equation for the drag coefficient is:

$C_{D}=\frac{D}{(1 / 2) \rho U^{2} \pi a^{2}}=\frac{24}{R_{p}}$,

with $R_{p}(=U d / v)$ based on diameter $(d=2 a)$ and kinematic viscosity $(v=\mu / \rho)$

The equations of Stokes have a serious defect since they result in streamlines that are symmetrical before and after the sphere and hence show no wake. This effect results from the elimination of vorticity convection through disregard of the inertia terms.

To find a more general expression for the drag of a sphere, Oseen $[9,10]$ proposed that a linearized form of the inertia terms should be kept in the distant field of flow. The Oseen correction to the Stokes drag coefficient leads to:

$C_{D}=\frac{24}{R_{p}}\left(1+\frac{3}{16} R_{p}\right)$

Behind a sphere, at $R_{p}=1$, the flow pattern given by Oseen's solution shows a clearly defined wake, but no evidence of an incipient vortex. This is not in observance with experiments and, in fact, for larger values of $R_{p}$, solutions based on perturbation techniques must not be expected to coincide with solutions yielded by the N.-S. equations (Illingworth [12]).

Nevertheless, the improvement in predictions resulting from the solution of Oseen motivated a series of workers to pursue higher-order corrections to Eq. (2). As stated by Weisenborn and Bosch [13], such studies further increase in importance upon the realization that drag estimates based on the Oseen approximation are in some cases related to the results based on the N.-S. equations.

Quoting Stewartson [14], Weisenborn and Bosch [13] mention that for large $R_{p}$, one-third of the Oseen drag on a sphere tends to the experimental values. The 3:1 ratio between Oseen estimates and experiments is observed for $R_{p}$ between 50 and 100,000. Weisenborn and Bosch [13] also mention that at infinite $R_{p}, C_{D}$ takes on the value 1.058 .

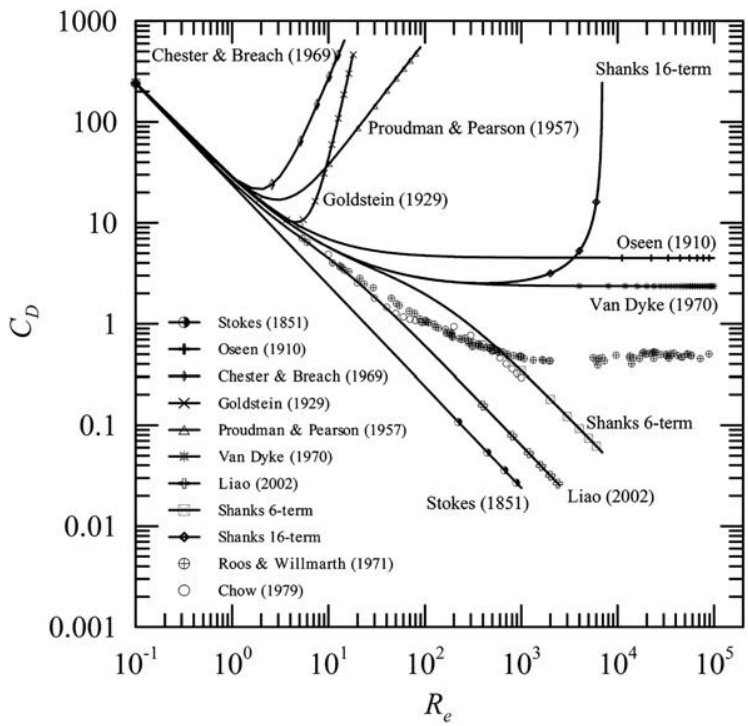

Fig. 1. Comparison between theories and the experimental data of Roos and Willmarth [6] and the numerical data of Chow [18].

\section{Series for the Oseen drag of a sphere}

This section shortly reviews the formulations of other authors for extensions of the Oseen equation.

The models discussed in this section are shown in advance in Fig. 1 so that all theoretical results can be appreciated relatively to each other. In addition to the solutions of Stokes and Oseen, Fig. 1 illustrates the models that will be discussed next, the models of Goldstein [5], Proudman and Pearson [15], Chester and Breach [16], Van Dyke [17] and Liao [1] as well as the experimental data (black points) of Roos and Willmarth [6] and the numerical data (small empty circles) of Chow [18] obtained through an application of Galerkin method to the full Navier-Stokes equations. Fig. 1 also shows the results obtained by application of Shanks transformations (6 and 16 terms respectively) to the solution of Goldstein [5].

Starting with Oseen's equation, Goldstein [5] proposed the drag of a sphere to be described according to the expression:

$$
\begin{aligned}
C_{D}=\frac{24}{R_{p}}( & +\frac{3}{16} R_{p}-\frac{19}{1280} R_{p}^{2}+\frac{71}{20480} R_{p}^{3} \\
& \left.-\frac{30179}{34406400} R_{p}^{4}+\frac{122519}{550502400} R_{p}^{5}\right) .
\end{aligned}
$$

This series is useful for $R_{p}$ up to 2 , but quickly diverges for $R_{p}$ larger than 5. Subsequently, Goldstein (1938) stated that "if the neglected terms in the equations of motion could be retained, only the first two terms in the expansion of $C_{D}$ would be unaltered".

Van Dyke [17] extended Eq. (3) to 24 terms. He also used a linear fractional transformation to exclude a singularity from the negative axis of $R_{p}$ and extend the convergence region of $C_{D}$ to infinity. However, his solution is only satisfactory to $R_{p}$ smaller than 5 .

Hunter and Lee [19] computed 66 terms of Goldstein's power series. They show that neither Padé approximants nor a Euler transformation formed from their solution gives good convergence. The asymptotic behavior of $C_{D}$ is suggested to proceed in powers of $R_{p}^{-2 / 3}$. Similar suggestion had already been advanced by Van Dyke [17].

To improve the previous results, some authors have applied perturbation methods directly to the N.-S. equations. Using Stokes variables for the inner flow and Oseen variables for the outer flow, Kaplun and Lagerstrom [20] were able to find a composite expansion uniformly valid in the entire flow domain. Their method, taken up by Proudman and Pearson [15] leads to:

$C_{D}=\frac{24}{R_{p}}\left(1+\frac{3}{16} R_{p}-\frac{9}{160} R_{p}^{2} \ln R_{p}\right)$.

A higher-order correction of Eq. (4) was advanced by Chester and Breach [16],

$C_{D}=\frac{24}{R_{p}}\left[1+\frac{3}{16} R_{p}-\frac{9}{160} R_{p}^{2}\left(\ln R_{p}+\gamma+\frac{2}{3} \ln 2-\frac{323}{360}\right)+\frac{27}{640} R_{p}^{3} \ln R_{p}\right]$

where $\gamma$ is the Euler constant.

Liao [1] notices that Eq. (4) only furnishes good results for $R_{p}<1$. In fact, the radius of convergence of Eq. (5) is shorter than that of Eq. (4).

Using the homotopy analysis method, Liao [1] proposed a solution to the problem of a viscous flow past a sphere in a uniform stream. The method is applied directly to the N.-S. equations and is observed to give good prediction for $R_{p}$ up to 30 .

\section{Rational fractions}

Shanks [4] developed a remarkable non-linear operation to transform divergent and slowly convergent series in rational fraction functions with improved convergences. One of the present authors (MDM), 
developed a special Mathematica ${ }^{\mathrm{TM}}$ package to construct such nonlinear transformation.

To establish an accurate approximation to $C_{D}$ from the few terms of Eq. (3) and increase its range of convergence, a generalized Shanks transformation can be used with the extra information that the drag coefficient is finite at infinite $R_{p}$.

The Shanks transformation of Goldstein series (Eq. (3)) with 6 polynomial terms gives

$C_{D_{S}}=\frac{1920\left(3696+1665 R_{p}+136 R_{p}^{2}\right)}{R_{p}\left(295680+77760 R_{p}+689 R_{p}^{2}\right)}$.

Eq. (6) follows the correction of Van Dyke [17] up to $R_{p}=10$, plunging next to unphysical values. For larger $R_{p}$ this equation does not agree with the data of Roos and Willmarth [6].

A further application of Shanks transformation with 16 polynomial terms to the series of Goldstein yields a very complex expression (not shown here). Results, however, are much worse than those provided by Eq. (6) (Fig. 1).

\section{Present proposition}

The difficulties illustrated by Fig. 1 have led authors to propose approximations for the estimation of $C_{D}$ from empirical or numerical data through regression techniques. Two recent articles by Cheng [2] and Almedeij [3] review respectively nine and seven different expressions for the prediction of $C_{D}$. These are discussed in the next section.

Here, to improve Eq. (6), we fit its coefficients directly to the experimental data of Roos and Willmarth [6] in the range $5.33<R_{p}<118,300$, to obtain

$C_{D_{P}}=\frac{777\left((669806 / 875)+(114976 / 1155) R_{p}+(707 / 1380) R_{p}^{2}\right)}{646 R_{p}\left((32869 / 952)+(924 / 643) R_{p}+(1 / 385718) R_{p}^{2}\right)}$.

Results given by Eq. (7) are shown in Fig. 2. Eq. (7) is based on a non-linear rational fraction transform of the series of Goldstein (Eq. (6)), thus establishing a physically meaningful form that can be used to fit the experimental data. Eq. (7) can be used in the interval $5.33<R_{p}<118,300$, with a standard deviation of 0.04 .

For low Reynolds numbers, in the range $0.1<R_{p}<10$, the expression of Shanks (1955), Eq. (6), should be used (see Fig. 1). Alternatively,

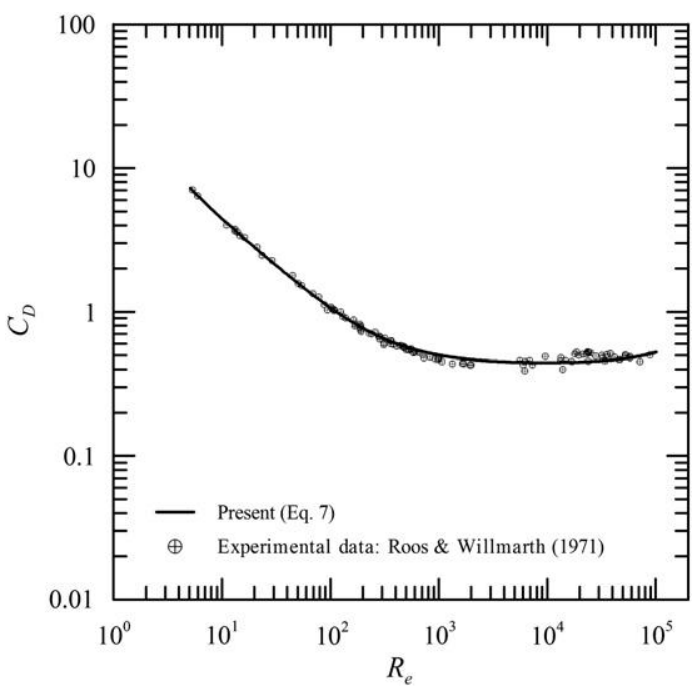

Fig. 2. Comparison between Eq. (7) the data of Roos and Willmarth [6].

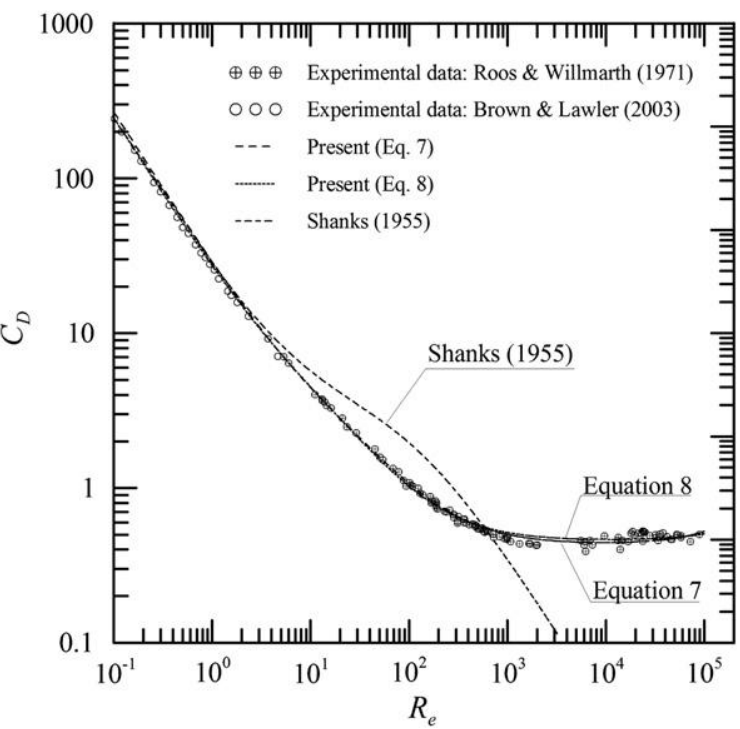

Fig. 3. Behavior of Eqs. (6), (7) and (8) against the data of Roos and Willmarth [6] and Brown and Lawler [7]

Eq. (7) can be improved for low $R_{p}$, provided that the experimental data of Brown and Lawler [7] are considered (Fig. 3).

The resulting expression is shown below (Eq. (8)),

$C_{D_{A}}=\frac{3808\left((1617933 / 2030)+(178861 / 1063) R_{p}+(1219 / 1084) R_{p}^{2}\right)}{681 R_{p}\left((77531 / 422)+(13529 / 976) R_{p}-(1 / 71154) R_{p}^{2}\right)}$.

This expression significantly improves the low- $R_{p}$ results, but furnishes slightly higher results of $C_{D}$ for very large values of $R_{p}$. Predictions furnished by Eqs. (6), (7) and (8) are shown in Fig. 3 and Table 1.

\section{Comparison with the $C_{D}$-expressions of other authors}

Eq. (7) here is compared with the expressions reported in Cheng [2]. These formulas are supposed to be very accurate and valid up to $R_{p}<10^{5}$. In addition to his own formulation, Cheng discusses the expressions originally presented by Almedeij [3], Brown and Lawler [7], Flemmer and Banks [21], Turton and Levenspiel [22], Concha and Barrientos [23], Clift et al. [24]. All expressions are presented in detail in Table 1 of Cheng [2].

The formulas of Cheng [2], Brown and Lawler [7], Clift et al. [24] and Turton and Levenspiel [22] bear great resemblance. They basically have the form,

$C_{D}=\frac{24}{R_{p}}\left(1+a R_{p}^{m}\right)+\frac{b}{1+c R_{p}^{-n}}$

Table 1

Comparison between results provided by Eqs. (6) through (8) and the experimental data.

\begin{tabular}{lcccc}
\hline$R_{p}$ & Eq. (7) & Eq. (8) & Eq. (6) & Experiments \\
\hline 0.1 & 269.02 & 245.85 & 244.47 & 242.63 \\
1 & 28.95 & 27.35 & 28.21 & 27.24 \\
11 & 4.17 & 4.20 & 5.44 & 4.30 \\
101 & 1.064 & 1.023 & 1.953 & 1.08 \\
1000 & 0.5016 & 0.5155 & 0.3446 & 0.478 \\
9620 & 0.4441 & 0.4645 & 0.0390 & 0.49 \\
100,000 & 0.5241 & 0.5055 & 0.00378 & 0.467 \\
\hline
\end{tabular}


where $a, b, c, m$ and $n$ assume different values according to the different authors.

Concha and Barrientos [23] write

$C_{D}=\frac{0.284153}{R_{p}^{2}}\left(1+\frac{\delta_{0}}{R_{p}^{1 / 2}}\right)^{2} \sum_{1}^{5} \beta_{i} R e^{i}$

where $\delta_{0}$ and the $\beta_{i}$ 's are constants adjusted from experimental data.

Flemmer and Banks [21] and Almedeij [3] make respectively

$C_{D}=\frac{24}{R_{p}} 10^{\alpha}, \alpha=d R_{p}^{p}-e R_{p}^{q}-\frac{f}{1+\log ^{2} R_{p}}$

$C_{D}=\left[\frac{1}{\left(\varphi_{1}+\varphi_{2}\right)^{-1}+\varphi_{3}^{-1}}+\varphi_{4}\right]^{1 / 10}$,

where, $\alpha$ and functions $\varphi_{i}$ are again determined from the experimental data.

The formula of Clift et al. [24] consists of ten stepwise approximations.

None of the above expressions consists of a rational fraction, such as expression (7). To compare the present results with other authors, we consider the absolute error, $C_{D \text { (literature) }}-C_{D \text { (present) }}$. Fig. 4 shows the deviations of Eq. (7) from the other formulations for $R_{p}$ ranging from 100 to 100,000 . (P.S.: Symbols here are only used to identify the different curves; they do not show the actual frequency of experimental data).

Notice that for low $R_{p}$, of the ordem of 100 , a large disagreement is observe between all correlations. In general, the formulations of Cheng [2] and Brown and Lawler [7] are close together and far from those of Clift et al. [24], Flemmer and Banks [21] and Concha and Barrientos [23]. The expression of Almedeij [3] has the most distinct behavior, approaching the correlation of Turton and Levenspiel [22] and the present correlation for $2.10^{4}<R_{p}<6.10^{4}$.

\section{Final remarks}

The present work has advanced a rational fraction (Eq. (7)) for the evaluation of the drag coefficient of a falling sphere that can be used in applications with $R_{p}$ in the range 5 to 100,000 . An alternative expression is also proposed, (Eq. (8)), to improve prediction for low $R_{p}$ in the interval $(0.1,10)$.

The basis for Eqs. (7) and (8) is the non-linear operation introduced by Shanks to transform divergent and slowly convergent series in rational fraction functions with improved convergences. Here, a Shanks transformation is applied directly to the data of Roos and Willmarth [6], to allow the range of validity of Eq. (7) to be expanded to 100,000 .

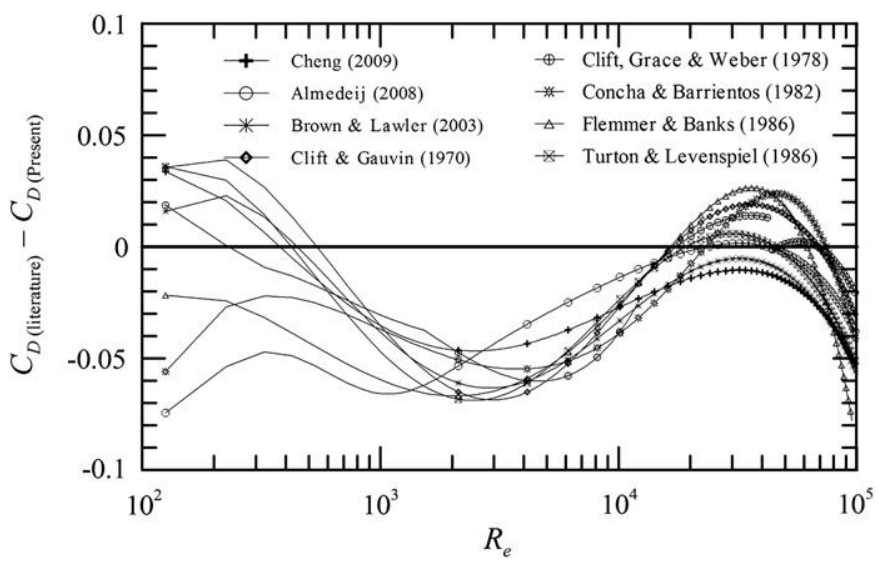

Fig. 4. Comparison between Eq. (7) and the formulation of other authors.
The proposed expressions are particularly very simple, so as to make their practical implementation an easy procedure.

\section{Acknowledgments}

MDM is thankful to the Brazilian National Research Council (CNPq) and the Brazilian National Institute of Standards (INMETRO) for the financial support to this research through Grant 563061/2010-3. APSF is grateful to the Brazilian National Research Council (CNPq) for the award of a Research Fellowship (Grant No 303982/2009-8). The work was financially supported by the Rio de Janeiro Research Foundation (FAPERJ) through Grant E-26/170.005/2008.

\section{References}

[1] S.-J. Liao, An analytic approximation of the drag coefficient for the viscous flow past a sphere, International Journal of Non-Linear Mechanics 37 (2002) 1-18.

[2] N.-S. Cheng, Comparison of formulas for drag coefficient and settling velocity of spherical particles, Powder Technology 189 (2009) 395-398.

[3] J. Almedeij, Drag coefficent of flow around a sphere: matching asymptotically the wide trend, Powder Technology 186 (2008) 218-223.

[4] D. Shanks, Non-linear transformation of divergent and slowly convergent sequences, Journal of Mathematical Physics 34 (1955) 1-42.

[5] S. Goldstein, The steady flow of viscous fluid past a fixed spherical obstacle at small Reynolds numbers, Proceedings of the Royal Society of London A 123 (1929) 225-235.

[6] F.W. Roos, W.W. Willmarth, Some experimental results on sphere and disk drag, AIAA Journal 9 (1971) 285-290.

[7] P.P. Brown, D.F. Lawler, Sphere drag and settling velocity revisited, Journal of Environmental Engineeering-ASCE 129 (3) (2003) 222-231.

[8] G.G. Stokes, On the effect of internal friction of fluids on the motion of pedulums, Transactions of the Cambridge Philosophical Society 9 (1851) 8-106.

[9] C.W. Oseen, Ueber die Stokessche Formel und die verwandte Aufgabe in der Hydrodynamik, Arkiv för Matematik, Astronomi och Fysik 6 (1910) 29.

[10] C.W. Oseen, Uber den Giiltigkeitsbereich der Stokesschen Widerstandsformel, Arkiv för Matematik, Astronomi och Fysik 9 (16) (1913) 1-15.

[11] M. Van Dyke, Perturbation methods in fluid mechanics, Parabolic Press, 1975

[12] C.R. Illingworth, Flow at small Reynolds number, in: L. Rosenhead (Ed.), Laminar boundary layers, Clarendon Press, Oxford, 1963.

[13] A.J. Weisenborn, B.I.M. Ten Bosch, Analytical approach to the Oseen drag on a sphere at infinite Reynolds number, SIAM Journal on Applied Mathematics 53 (1993) 601-620.

[14] K. Stewartson, On the steady flow past a sphere at high Reynolds number using Oseen's approximation, Philosophical Magazine 1 (1956) 345-354.

[15] I. Proudman, J.R. Pearson, Expansion at small Reynolds number for the flow past a sphere and a circular cylinder, Journal of Fluid Mechanics 2 (1957) 237-262.

[16] W. Chester, D.R. Breach, On the flow past a sphere at low Reynolds number, Journal of Fluid Mechanics 37 (1969) 751-760.

[17] M.Van Dyke, Extension of Goldstein's series for the Oseen drag of a sphere, Journal of Fluid Mechanics 44 (1970) 365-372.

[18] C.-Y. Chow, An introduction to computational fluid mechanics, Seminole Publishing Company, 1979.

[19] C. Hunter, S.M. Lee, The analytic structure of Oseen flow past a sphere as a function of Reynolds number, SIAM Journal on Applied Mathematics 46 (1986) 978-999.

[20] S. Kaplun, P.A. Lagerstrom, Asymptotic expansions of Navier-Stokes solutions for small Reynolds numbers, Journal of Mathematics and Mechanics 6 (1957) 585-593.

[21] R.L.C. Flemmer, C.L. Banks, On the drag coefficient of a sphere, Powder Technology 48 (3) (1986) 217-221.

[22] R. Turton, O. Levenspiel, A short note on the drag correlation for spheres, Powder Technology 47 (1) (1986) 83-86.

[23] F. Concha, A. Barrientos, Settling velocities of particulate systems, 3. Power-series expansion for the drag coefficient of a sphere and prediction of the settling velocity, International Journal of Mineral Processing 9 (2) (1982) 167-172.

[24] R. Clift, J.R. Grace, M.E. Weber, Bubbles, drops, and particles, Academic Press, New York, 1978. 